\title{
Laudatio zur Verleihung der Ehrenmitgliedschaft der DEGUM an Prof Dr. Eberhard Merz
}

Die Deutsche Gesellschaft für Ultraschall in der Medizin (DEGUM) verleiht Herrn Prof. Dr. Eberhard Merz die Ehrenmitgliedschaft der Gesellschaft. Diese Ehrung ist Ausdruck der Würdigung der jahrzehntelangen Verdienste von Eberhard Merz für den Ultraschall.

Eberhard Merz ist eines der „Urgesteine“ der DEGUM und einer der wesentlichen Wegbereiter des Ultraschalls in der Gynäkologie und Geburtshilfe. Er ist einer der klinischen Pioniere auf dem Gebiet des 3-D-Ultraschalls im Bereich der Gynäkologie, Geburtshilfe und der Mammasonografie. Unter anderem organisierte er den ersten Weltkongress für 3-D-Sonografie. Er gehört zu denjenigen, die noch das gesamte Fachgebiet wissenschaftlich erkundet haben, in der Geburtshilfe sind z.B. vielen Kollegen die verschiedenen Normkurven „nach Merz“ ein Begriff.

\section{Einige Stationen aus seinem Curriculum vitae}

$\nabla$

- 1969 - 1976 Studium der Biologie, Physik, Chemie und Medizin in Tübingen, Marburg und Kiel

- 1977 Promotion

- 1985 Facharzt für Frauenheilkunde und Geburtshilfe

- 1988 Habilitation

> 1989 DEGUM Stufe III Sektion Gynäkologie und Geburtshilfe

> 1995 Außerplanmäßiger Professor

- 1997 Fakultative Weiterbildung (spezielle operative Gynäkologie, spezielle Geburtshilfe und Perinatalmedizin, gynäkologische Endokrinologie und Reproduktionsmedizin)

> 2006 Schwerpunktbezeichnung Gynäkologische Onkologie

- 2010 DEGUM Stufe III Mammasonografie

Von 2000 - 2010 war er Mitglied des Vorstands der DEGUM, von 2006 bis 2008 war er Präsident der DEGUM, von 2008 bis 2010 Alt-Präsident. Vom 20.-23. Oktober 2010 war er Kongresspräsident des 34. Dreiländertreffens von DEGUM, ÖGUM und SGUM in Mainz.

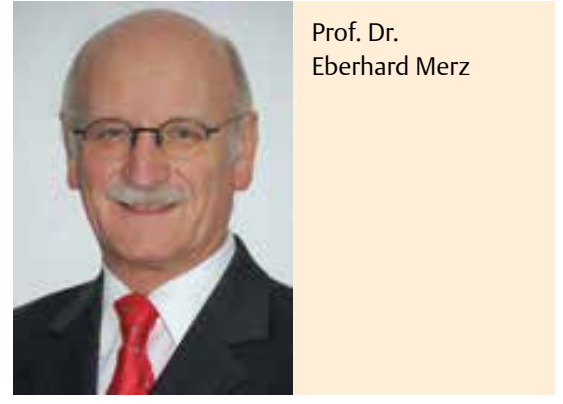

Gleichzeitig prägte er auch andere internationale Fachgesellschaften: er war Executive Board Member der ISUOG, aktuell ist er Vorsitzender der Fetal Medicine Foundation (FMF) Deutschland.

Eberhard Merz ist durch eine umfangreiche wissenschaftliche Arbeit gekennzeichnet, die auch mit zunehmendem Alter nicht weniger wird. So ist er Autor bzw. Ko-Autor von mehr als 240 wissenschaftlichen Publikationen. Auf dem ISUOG Weltkongress 2014 in Barcelona wurden durch Eberhard Merz wissenschaftliche Beiträge im Rahmen des Kongressprogramms angemeldet und dort vertreten. Er ist Herausgeber von 6 Ultraschallehrbüchern der Gynäkologie und Geburtshilfe, wobei 2 davon in 7 Sprachen übersetzt wurden.

Eberhard Merz ist unter anderem Mitherausgeber der Zeitschrift, Ultraschall in der Medizin' (seit 1998), die aktuell mit einem Impact von $>4$ eine der hoch dotierten wissenschaftlichen Zeitschriften mit Schwerpunkt Ultraschall ist.

Neben allen wissenschaftlichen Meriten ist Eberhard Merz aber auch ein Mensch geblieben, der neugierig auf die Welt, die Menschen und auch neue Techniken ist. Diese Eigenschaft stellte er beim Referentenabend in Davos eindrücklich unter Beweis!

Wir wünschen ihm und seiner Familie, dass dieses immer so bleibt!

Berlin, 17. November 2015

Kai-Sven Heling 